\title{
The GlucoCoper - a Tool for the Assessment of Coping Mechanisms
}

\author{
Sanjay Kalra, ${ }^{1}$ Yatan Pal Singh Balhara, ${ }^{2}$ Komal Verma ${ }^{3}$ and Bharti Kalra ${ }^{4}$ \\ 1. Department of Endocrinology, Bharti Hospital, Karnal, India; 2. National Drug Dependence Treatment Centre (NDDTC) \& Department of \\ Psychiatry, All India Institute of Medical Science (AIIMS), New Delhi, India; 3. Department of Behavioural and Allied Sciences, Amity University, \\ Rajasthan, India; 4. Department of Obstetrics and Gynaecology, Bharti Hospital, Karnal, India
}

$\mathrm{D}$ labetes distress - commonly encountered as a part of life with diabetes mellitus - can be effectively managed by coping skills training. systematic assessment and documentation of the existing coping skills (and lack thereof) is a pre-requisite for planning such coping skills training. A six-item tool, the GlucoCoper - which assesses two negative (resistance, blame) and four positive (acceptance, optimism, planning and action) coping mechanisms - was administered to English-literate adult participants with type 2 diabetes (T2DM) at an endocrine centre in India. Each item was graded on a 10-point Likert scale. The GlucoCoper was administered to 338 participants. The tool demonstrated high internal validity. Total score on the GlucoCoper correlated with fasting and postprandial glucose. The GlucoCoper is a valid tool which can be used to assess and rate coping in persons with T2DM. It has potential in helping plan, deliver and monitor coping skills training in persons with diabetes distress.

\section{Keywords}

Depression, diabetes distress, patientcentred care, patient-reported outcomes psychosocial aspects, type 2 diabetes

Disclosure: Sanjay Kalra, Yatan Pal Singh Balhara, Komal Verma and Bharti Kalra have nothing to declare in relation to this article.

Review Process: Double-blind peer review.

Compliance with Ethics: Ethical approval and patient consent were received for this study.

Authorship: All named authors meet the International Committee of Medical Journal Editors (ICMJE) criteria

for authorship of this manuscript, take responsibility for the integrity of the work as a whole, and have given final approval to the version to be published.

Open Access: This article is published under the Creative Commons Attribution Noncommercial License, which permits any non-commercial use, distribution, adaptation and reproduction provided the original author(s) and source are given appropriate credit. (C) The Authors 2018.

Received: 10 January 2018

Accepted: 14 February 2018

Citation: European Endocrinology. 2018;14(1):52-55

Corresponding Author: Sanjay Kalra, Bharti Hospital, Karnal 132001, India. E: bridekn|@gmail.com

Support: No funding was received in

the publication of this article.
Diabetes distress (DD) is a frequently documented aspect of living with diabetes. ${ }^{1}$ DD is defined as an emotional state characterised by extreme apprehension, discomfort or dejection due to perceived inability in coping with the challenges and demands of living with diabetes. ${ }^{2}$ The presence of DD impairs psychosocial functioning and prevents the achievement of optimal biomedical health. It is important, therefore, to address DD in a timely and proactive manner. In fact, DD can be effectively managed by non-pharmacological means including education, support and counselling. One specific technique to manage DD is coping skills training (CST).3., The systematic assessment and documentation of existing coping skills (or lack thereof) is a pre-requisite for planning such intervention. ${ }^{2}$

Diabetes distress can be assessed using the existing validated tools such as the Diabetes Distress Scale (DDS). ${ }^{5-8}$ Specific versions of this instrument are available for use in type 1 diabetes, type 2 diabetes (T2DM), parents and caregivers. It is recommended that if significant DD is present, or anticipated, coping mechanisms should be analysed. The assessment of coping mechanisms may precede, accompany or follow the measurement of DD. It is debatable as to what should be analysed first. In fact, a systematic assessment of coping skills may actually help predict diabetes distress at a later stage. The assessment of coping skills is, therefore, suggested as a primary investigation for DD treatment as well as prevention. ${ }^{2}$

While the DDS provides a quantitative assessment of the degree of DD, its utility as a constructive platform to build upon therapeutic interventions or plan further management is restricted by its structure. The DDS is not designed to identify specific shortcomings or weakness in coping styles that can be targeted to improve the emotional health of patients with DD. One way of achieving this goal is to analyse the coping skills of patients with diabetes mellitus.

The ability to cope with diabetes is a tool, as well as a target, of effective diabetes care. The need for efficient coping skills is clearly mentioned in the current position statements from leading professional organisations." A recent meta-analysis and review has shown that good coping skills and self-efficacy correlated strongly with dietary adherence, which itself strongly relates to improved glycaemic control. ${ }^{10}$ Significant relationships of glycated haemoglobin (HbA1C) have been reported with self-care activities, coping styles and social support in adults with T2DM.11 Perceived stress and coping skills vary with gender and age. Males with T2DM living with their spouse were found to be strongly dependent on support from the spouse. ${ }^{12}$

A recent regression analysis on 142 adult subjects with T2DM showed that DD is significantly related to medication adherence and HDA1c. Self-efficacy and perceived control are also independently associated with both these variables. Mediation analyses have demonstrated a significant indirect effect on DD and medication adherence through perceived control and self-efficacy. ${ }^{13}$ 
Thus, coping or control skills in diabetes can be studied as a positive corollary of glycaemic control, rather than focusing on the negative concept of 'distress'.

Specific coping mechanisms have been linked with glycaemic control in persons with diabetes. Avoidance coping strategies are negative-emotion focused and are associated with negative psychological outcomes, poor treatment adherence and poor metabolic control..$^{14}$ At the same time, acceptance coping strategies, which are problem-focused and positiveemotion focused, are associated with improved glucose control. ${ }^{15}$ Acceptance and commitment therapy have been found to improve diabetes-related outcomes as well. ${ }^{16}$

While validated psychometric tools are available to measure coping skills, they are generic in nature and do not specifically meet the needs of persons with diabetes. Also, there is no tool which can simultaneously serve a dual purpose of screening/identification and intervention/ treatment. We therefore felt the need to develop a tool which could identify the coping styles and mechanisms of persons with diabetes and facilitate the institution of appropriate management strategies.

Based upon clinical experience and discussion, a screening tool called the GlucoCoper was developed. Discussions among a multi-professional team, experienced in the care of patients with diabetes, led to the crystallization of six common coping mechanisms. This list was deemed comprehensive enough to cover all coping styles and yet short enough to serve as a convenient screening tool. The Glucocoper was designed to be visually appealing, simple to understand and easy to administer. The tool was pre-tested among patients with T2DM attending the endocrine clinic. It is a six-item tool that analyses six coping styles (two negative, four positive). These are: negativity, blame, acceptance, optimism, planning and action. The respondents answered six questions on a 1-10 visual 'thermometer-lookalike' 'cope-meter' scale. The responses provided a score for each individual coping strategy employed by the respondents. The tool then provides a total score, a positive scale score and a negative scale score - the scores of the four positive coping mechanisms (acceptance, optimism, planning and action) are added to calculate the positive scale score and the scores of the two negative coping methods (resistance and blame) are added to form the negative scale score. The negative scale score is subtracted from the positive scale score to calculate the total coping score. The possible range of scores for each individual coping mechanism is 1-10; the total positive scale score ranges from 4-40; the negative scale score ranges from 2-20; the total score ranges from -16 to 38 . These three scores offer information on the current coping styles and help identify areas for intervention. The values obtained with the Glucocoper can be charted on graphs to allow a reader-friendly impression of changes in coping styles over time (Figure 1).

\section{Aims and objectives}

The Glucocoper is a novel tool aimed at the screening and assessment of $\mathrm{DD}$ and coping mechanisms among persons with diabetes mellitus. The current study aimed to explore the utility and validity of the GlucoCoper as a screening and assessment tool for DD and coping mechanisms in patients with T2DM.

\section{Material and methods}

The study was conducted at an endocrine centre in northern India. The GlucoCoper was administered to 338 consecutive patients with T2DM who presented to the outdoor endocrine clinic for diabetes management. Inclusion criteria included age (more than 18 years), a confirmed diagnosis of T2DM, literacy in English and willingness to

\section{Figure 1: The GlucoCoper tool}

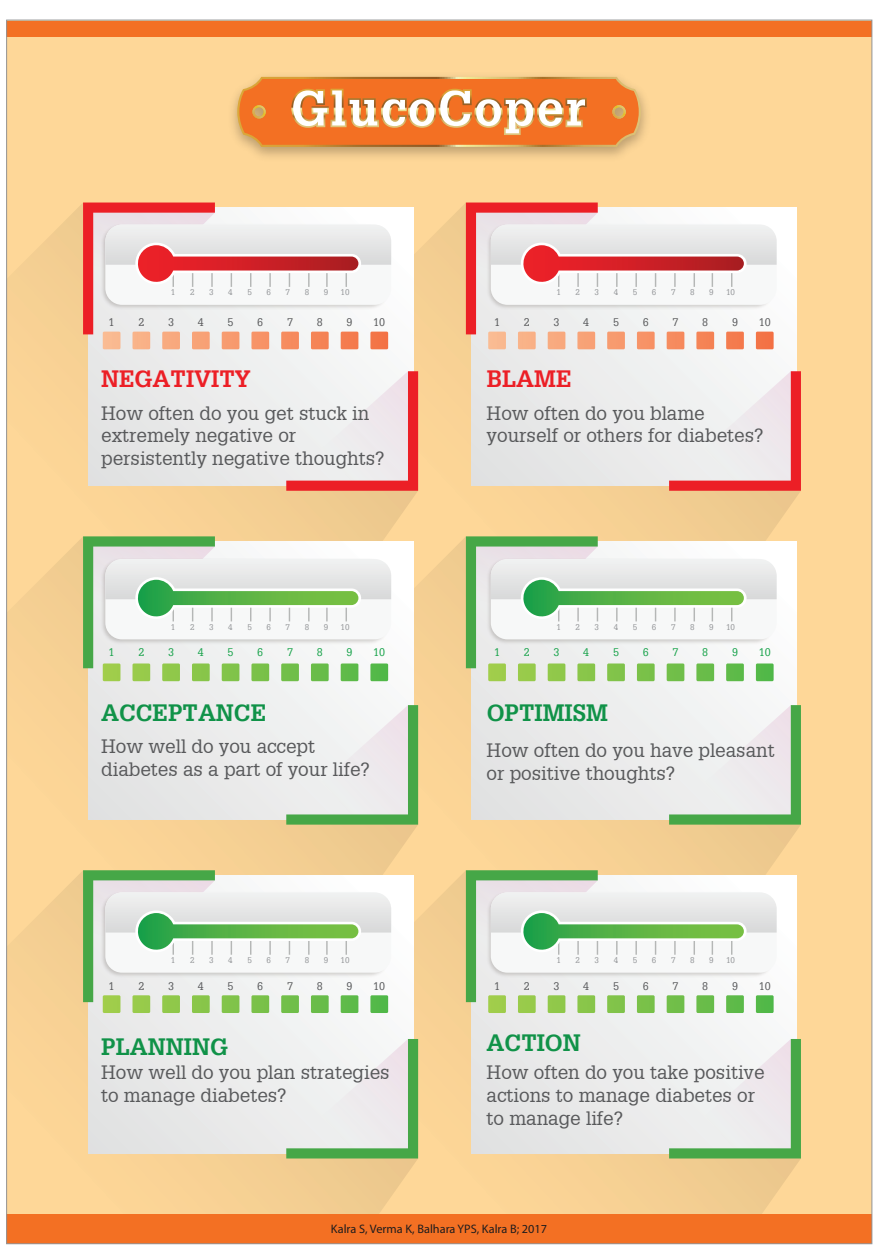

provide informed consent. Exclusion criteria included patients with a diagnosis of gestational diabetes, antenatal women, patients with type 1 diabetes and those who required in-patient treatment. The Glucocoper was administered by a trained and experienced diabetes counsellor. Results were analysed using Statistical Package for the Social Sciences (SPSS) version 16.0.

\section{Results}

The baseline characteristics of the study subjects are shown in Table 1. The scores of the GlucoCoper - positive, negative and total scale scores - are listed in Table 2.

The total score was found to have a significant and strong positive correlation with the positive scale and each positive coping strategy $(p<0.001$ for all). The total score also exhibited significant and strong negative correlation with both the negative scale and with each individual negative coping mechanism ( $p<0.001$ for all). Total scores correlated significantly with fasting plasma glucose (FPG) and postprandial plasma glucose (PPG) ( $p<0.001)$, but not with age, HbA1c, duration of diabetes, number of injections per day or presence/absence of complications. A statistically significant correlation was also noted with number of tablets of oral hypoglycaemic agents $(\mathrm{OHA})$ consumed per day $(\mathrm{p}<0.05)$.

The positive scale score correlated strongly with each of the four positive coping mechanisms ( $p<0.001)$. Similarly, the negative scale score correlated negatively with both of the negative coping mechanisms $(p<0.05)$. A significant correlation was found between the positive scale and number of OHA tablets consumed per day $(p<0.001)$, while 
Table 1: Baseline characteristics of participants

\begin{tabular}{|l|l|l|l|l|l|l|}
\hline & Unit & \multicolumn{2}{l}{ Minimum } & Maximum & Mean & Standard Deviation \\
\hline Age & Years & 22.0 & 88.00 & 50.19 & 12.20 \\
\hline HbA1C & $\%$ & 5.30 & 17.00 & 8.72 & 2.10 \\
\hline FPG & $\mathrm{mg} \%$ & 57.00 & 397.00 & 156.41 & 60.54 \\
\hline PPG & $\mathrm{mg} \%$ & 101.00 & 600.00 & 227.56 & 86.39 \\
\hline $\begin{array}{l}\text { Duration of } \\
\text { diabetes }\end{array}$ & Years & 2.00 & 32.00 & 16.11 & 7.32 \\
\hline
\end{tabular}

$F P G$ = fasting plasma glucose $;$ HbA1C = glycosylated haemoglobin; $P P G=$ postprandial g/ucose.

Table 2: Baseline GlucoCoper score of participants

\begin{tabular}{|l|l|l|l|l|}
\hline & Minimum & Maximum & Mean & Standard Deviation \\
\hline Resistance & 2.00 & 10 & 5.7189 & 1.81512 \\
\hline Blame & 1.00 & 10 & 5.2130 & 3.15319 \\
\hline Acceptance & 2.00 & 8 & 5.8225 & 1.45898 \\
\hline Optimism & 2.00 & 9 & 6.1834 & 1.45213 \\
\hline Planning & 1.00 & 9 & 5.0503 & 2.09998 \\
\hline Action & 1.00 & 9 & 6.2160 & 1.71849 \\
\hline Negative scale score & 3.00 & 18 & 10.9320 & 3.87851 \\
\hline Positive scale score & 6.00 & 33 & 23.2722 & 5.18299 \\
\hline Total score & -6.00 & 29 & 12.3402 & 6.26560 \\
\hline
\end{tabular}

a negative correlation was demonstrated between positive scale score and FPG, as well as PPG values $(p<0.001)$. The negative scale score correlated significantly with duration of diabetes $(p<0.05)$. A positive correlation was also observed between negative scale and one positive coping mechanism, i.e. action $(p<0.01)$.

The correlation of baseline characteristics such as age, $\mathrm{HbA1c}$, FPG, PPG, duration of diabetes, number of injections per day, number of OHA tablets consumed per day and complications are shown in Table 3. Significant gender differences were observed in all the coping skills except blame $(p<0.05)$. Females scored higher than males on acceptance, optimism, planning and action. Males reported higher scores for negativity than females.

The relationship between coping skills and other variables was also assessed. After a frequency distribution analysis, retinopathy and hypertension were selected for analysis as a balanced proportion of cases were found in each of their categories. This allowed the assessment of coping styles in relation to presence (or absence) of microvascular and macrovascular complications. The t test revealed no significant difference in the six coping skills between the two groups. The $t$ test revealed that participants with hypertension had higher scores in planning and action than those living with diabetes without hypertension. There were no significant differences in other coping skills between the two groups.

Logistic regression analysis was performed to understand the relative contribution of various study variables to the total score on the GlucoCoper scale. Out of the 13 exploratory variables, only two were found to be significant - the presence of hypertension $(p=0.046)$ and the presence of neuropathy $(p=0.012)$.

Logistic regression analysis was also carried out to understand the relative contribution of various study variables to the positive scale score. Besides the presence of hypertension $(p=0.046)$ and neuropathy $(p=0.012)$, number of tablets consumed per day $(p=0.003)$ was also found to be a significant predictor.

\section{Discussion}

Diabetes distress is a well-characterised emotional state that is commonly encountered among persons living with diabetes. DD can be managed by non-pharmacological means. ${ }^{2}$ One of the pillars of DD mitigation is CST. ${ }^{5}$ Assessment of current coping skills is a pre-requisite to effective CST. While validated generic scales are available for the assessment of how well a patient is coping, there is no valid tool which specifically explores coping skills in patients with diabetes.

The GlucoCoper is designed as a screening, rating, educational and monitoring tool. It simplifies and categorises styles of coping into two negative (negativity, blame) and four positive (acceptance, optimism, planning and action) mechanisms. This helps both patients and providers understand various methods of coping with diabetes and allows them to focus on improving their coping skills.2

The single centre nature of this research and the lack of comparison with other diagnostic tools such as the DDS are limitations that need to be addressed in future studies. However, the subjects included in the current study are representative of the heterogeneity that T2DM presents with. Some of the strengths of the study are the wide range of age, near equal inclusion of both genders, wide spectrum of duration of diabetes and presence of patients with varied levels of glycaemic control and complications. This allows easy extrapolation of the findings to realworld clinical practice.

The Glucocoper scores tend to fall with rise in glucose levels. This is a significant association with a possible bidirectional relationship. High ambient glucose levels may be associated with a decline in coping skills and with poor coping style there can be poorer glycaemic control. It is important to note, however, that we did not observe any correlation of GlucoCoper scores with HbA1C levels. This suggests that current or ambient glucose levels, rather than preceding glycaemic control, are more important and closely associated with ability to cope in diabetes. The number of tablets consumed daily also correlated positively with coping, suggesting that patients who willingly accepted a higher pill burden were likely to cope better with their condition.

The unexpected positive correlation between negative scale and the 'action' coping skill deserves attention. It is possible that patients who exhibit both negativity and blame score low on acceptance, optimism and planning. However, this does not prevent them from taking action to manage their diabetes. Negativity was more marked in older patients, while those with a longer history of diabetes were better at planning (though they scored higher on blame as well). It may be hypothesised that older patients find it more difficult to be optimistic, while those with a longer duration of illness gradually learn how to plan their self-care.

Number of tablets consumed per day correlated strongly with positive coping mechanisms, but number of injections administered per day did not. It must be noted that a higher number of injections per day was found to correlate with poor glycaemic control (HDA1C, FPG and PPG), longer duration of diabetes and greater number of complications. This implies that intensive insulin regimens (with greater frequency of injections) are prescribed, or accepted, only by patients with severe hyperglycaemia or significant complications. These confounding factors may explain the lack of significant association noted between frequency of injection and coping skills. 
Table 3: Correlation of variables with GlucoCoper item score

\begin{tabular}{|c|c|c|c|c|c|c|c|c|c|}
\hline & Resistance & Blame & Acceptance & Optimism & Planning & Action & $\begin{array}{l}\text { Negative Scale } \\
\text { Score }\end{array}$ & $\begin{array}{l}\text { Positive Scale } \\
\text { Score }\end{array}$ & Total Score \\
\hline Age & $0.109 * \star$ & & & & & & & & \\
\hline $\mathrm{HbA} 1 \mathrm{C}$ & & & & $-0.136^{\star}$ & & & & & \\
\hline FPG & $0.159 * *$ & & $-0.182 * \star *$ & $-0.258 * * *$ & & $-0.244^{* * *}$ & & $-0.234 * * *$ & -0.195 \\
\hline PPG & $0.184^{* *}$ & & $-0.161^{*}$ & $-0.253^{\star *}$ & & $-0.201 * * *$ & & $-0.201 * * *$ & $-0.187^{* *}$ \\
\hline Number of injections/day & $0.154^{* *}$ & & & & & & & & \\
\hline Number of tablets & & $0.137 * *$ & $0.163^{*}$ & $0.146^{*}$ & $0.151^{*}$ & $0.183^{* * *}$ & & $0.209 * * *$ & $0.114^{*}$ \\
\hline \multicolumn{10}{|l|}{ Total complications } \\
\hline Negative scale score & $0.596^{*}$ & $0.887^{*}$ & & & & $0.110^{* *}$ & & & $-0.565^{\star \star}$ \\
\hline Positive scale score & $-0.127^{*}$ & $0.154^{* *}$ & $0.747 * * *$ & $0.761^{* * *}$ & $0.737 * * *$ & $0.837^{* * *}$ & & & $0.786^{* *}$ \\
\hline
\end{tabular}

*significant at $p<0.05 ; * *$ significant at $p<0.01 ; * * *$ significant at $p<0.001$. Only significant correlations are listed in table. FPG = fasting-plasma glucose; HbA1C $=$ glycosylated haemoglobin; $P P G=$ postprandial g/ucose.

The logistic regression analysis results suggested that the total, positive scale and negative scale scores on the Glucocoper could not be explained on the basis of the usual correlates of diabetes that were included in the current study. This has important implications, as DD might be a construct that depends on additional factors beyond the ones measured in the current study. These may include social factors such as socioeconomic status and family support.10,12 We included most of the commonly assessed variables related to diabetes. This information translates into the fact that many of these variables cannot be used as proxy to understand DD. DD, therefore, is a construct that needs to be assessed separately in addition to the other variables associated with diabetes. The GlucoCoper score thus gains value as a separate 'vital sign' for diabetes assessment and monitoring. ${ }^{6,17}$

\section{Summary}

The Glucocoper is a validated tool to assess coping mechanisms in people with T2DM. Various factors including age, current glycaemic levels and duration of diabetes may influence coping mechanisms. The Glucocoper is a simple, easy to administer, patient- and professionalfriendly tool which helps quantify specific negative and positive coping mechanisms. The significant positive correlation of the overall coping score with positive scale score and with each individual positive coping skill, the negative correlations with negative scale score and negative coping skills, give strength to the psychometric properties of the Glucocoper. The internal validity of the scale allows it to be used as a screening tool for dysfunctional coping skills. The Glucocoper results can also be used as a template upon which to build interventions for individualised strategies for CST.
1. Fisher L, Gonzalez JS, Polonsky WH. The confusing tale of depression and distress in patients with diabetes: a call for greater clarity and precision. Diabet Med. 2014;31:764-72.

2. Kalra S, Verma K, Singh Balhara YP. Management of diabetes distress. J Pak Med Assoc. 2017;67:1625-7.

3. Grey $\mathrm{M}$, Boland EA, Davidson M, et al. Coping skills training for youth with diabetes mellitus has long-lasting effects on for youth with diabetes mellitus has long-lasting
metabolic control and quality of life. J Pediatr. metabolic control and

4. Kalra S, Kalra B. Mishti copes with diabetes: A pragmatic approach to coping skills training. I Soc Health Diabetes. 2017;5:1-2

5. Behavioral Diabetes Institute. Scales and Measures. Available at: http://behavioraldiabetes.org/scales-and-measures/ (accessed 1 January 2018)

6. Polonsky WH, Fisher L, Earles J, et al. Assessing psychosocial distress in diabetes: development of the diabetes distress scale. Diabetes Care. 2005;28:626-31.
7. Fisher L, Hessler DM, Polonsky WH, Mullan J. When is diabetes distress clinically meaningful?: establishing cut points for the Diabetes Distress Scale. Diabetes Care. 2012;35:259-64.

8. Fisher L, Glasgow RE, Mullan JT, Skaff MM, Polonsky WH. Development of a brief diabetes distress screening instrument. Ann Fam Med. 2008;6:246-52.

9. Powers MA, Bardsley J, Cypress M, et al. Diabetes selfmanagement education and support in type 2 diabetes: a joint management education and support in type 2 diabetes: a joint position statement of the American American Association or Diabetes Educators, and the Academy

. Brown SA, García AA, Brown A, et al. Biobehavioral

Brown SA, García AA, Brown A, et al. Biobehavioral
determinants of glycemic control in type 2 diabetes: determinants of glycemic control in type 2 diabetes: a 2016;99:1558-67.

11. Shayeghian Z, Aguilar-Vafaie ME, Besharat MA, et al. Self-care activities and glycated haemoglobin in Iranian patients with type 2 diabetes: can coping styles and social support have a buffering role? Psychol Health. 2015;30:153-64.
12. Hara Y, Hisatomi M, Ito $\mathrm{H}$, et al. Effects of gender, age, family support, and treatment on perceived stress and coping of patients with type 2 diabetes mellitus. Biopsychosoc Med. 2014;8:16.

13. Gonzalez JS, Shreck E, Psaros C, Safren SA. Distress and type 2 diabetes-treatment adherence: a mediating role for perceived control. Health Psychol. 2015;34:505-13.

14. Jaser SS, White LE. Coping and resilience in adolescents with type 1 diabetes. Child Care Health Dev. 2011; 37:335-42.

15. Huang CY, Lai HL, Lu YC, et al. Risk factors and coping style affect health outcomes in adults with type 2 diabetes. Biol Res Nurs. 2016;18:82-9.

16. Shayeghian $Z$, Hassanabadi $H$, Aguilar-Vafaie ME, et al. A randomized controlled trial of acceptance and commitment therapy for type 2 diabetes management: the moderating role of coping styles. PloS One. 2016;11:e0166599.

17. Kalra S, Verma K, Balhara YP. The sixth vital sign in diabetes. J Pak Med Assoc. 2017:67:1775-6. 